\title{
The First Presentation of Localized Scleroderma at Birth: Scleroderma as a Differential Diagnosis of Congenital Skin Lesion
}

\author{
Elmira Hajiesmaeil Memar ${ }^{1,2}$ \\ Vahid Ziaee ${ }^{1,2,4,5}$ \\ 1 Department of Pediatrics, Tehran University of Medical Sciences,
Tehran, Iran
${ }^{2}$ Children's Medical Center, Pediatric Center of Excellence, Tehran,
Iran
${ }^{3}$ Department of Pathology, Tehran University of Medical Sciences,
Tehran, Iran
4 Pediatric Rheumatology Society of Iran, Tehran, Iran
5 Pediatric Rheumatology Research Group, Rheumatology Research
Center, Tehran University of Medical Science, Tehran, Iran
}

J Child Sci 2022;12:e1-e4.

\author{
Mohammad-Hassan Moradinejad ${ }^{2,4}$
}

\begin{abstract}
Keywords

- localized scleroderma

- linear scleroderma

- diagnosis

- early infancy

- morphea

Localized scleroderma is an uncommon autoimmune disease characterized by fibrosis of the skin and underlying tissue without involvement of blood vessels or internal organs. It usually affects children during later childhood, and early presentation of localized scleroderma during infancy is rare. In the current study, we report a child with localized scleroderma-related presentations occurring at birth. A 2-day-old male neonate presented with a firm, erythematous, and slightly pigmented plaque on his left thigh, leading to a change in the diameter of the affected foot and contracture of the left knee. At the age of 7 months, he was referred to our rheumatology clinic with normal growth and development. Laboratory studies, including urine and blood high-performance liquid chromatography assay, antinuclear antibodies, antitopoisomerase I, and rheumatic factor, were in the normal range. No signs of ocular involvement were noted during ophthalmological consultation. Skin biopsy showed mild acanthosis and collagen bundles, which replaced the fat around the sweat glands. A final diagnosis of localized scleroderma was made. Treatment was started with oral prednisolone, oral methotrexate (MTX), and colchicine. The skin lesion stopped progressing after 3 months of treatment. Steroid was then tapered over 6 months, while MTX and colchicine were continued for 2 years. Localized scleroderma during early infancy is a rare disease, but it should be considered as a differential in infants with erythematous and firm lesions on their body at birth because early treatment can prevent future complications.
\end{abstract}

\begin{abstract}
Address for correspondence Vahid Ziaee, MD, Division of Pediatric Rheumatology, Children's Medical Center, No. 62, Dr. Gharib Street, Keshavarz Boulevard, Tehran 14194, Iran (e-mail: Ziaee@tums.ac.ir).
\end{abstract}

received

March 8, 2021

accepted after revision

November 13, 2021
DOI https://doi.org/

10.1055/s-0041-1741055.

ISSN 2474-5871. (c) 2022. The Author(s).

This is an open access article published by Thieme under the terms of the Creative Commons Attribution License, permitting unrestricted use, distribution, and reproduction so long as the original work is properly cited. (https://creativecommons.org/licenses/by/4.0/)

Georg Thieme Verlag KG, Rüdigerstraße 14, 70469 Stuttgart, Germany 


\section{Introduction}

Scleroderma is a group of autoimmune diseases involving the connective tissue, characterized by excessive deposition of collagen in the skin, internal organs, and blood vessels. ${ }^{1}$ Scleroderma includes two forms of the disease: systemic sclerosis, characterized by skin, arteries, and visceral fibrosis, and localized scleroderma characterized by fibrosis of the skin and underlying tissue without involvement of blood vessels or internal organs. ${ }^{2}$ Although localized scleroderma is uncommon, it is more common in children as compared with systemic sclerosis. ${ }^{3}$ In both adults and children, localized scleroderma is more frequent among women with a female-to-male ratio of $2: 1{ }^{4}$

Localized scleroderma, also called "morphea," usually manifests as an erythematous patch or firm, waxy-like patch surrounded by a lilac ring, or both in an active stage of the disease. The inactive stage of morphea is characterized by skin/subcutaneous tissue atrophy and hypo- or hyperpigmented patches.

Morphea has a different skin involvement pattern from systemic sclerosis and has been classified in several subgroups based on the pattern and depth of the lesions. Peterson et $\mathrm{al}^{5}$ described the traditional classification containing five subclassifications: plaque morphea, bullous morphea, generalized morphea, linear morphea, and deep and pansclerotic morpheae. The most common subtype in children is linear scleroderma, with the prevalence 50 to $60 \%$ in children with localized scleroderma. ${ }^{2}$ Linear scleroderma, characterized by a linear stick involvement of skin, subcutaneous fat, and often, the underlying tissues, presents as a single unilateral band on the extremities (with legs as the most common sites of involvement), trunk, or head. ${ }^{6,7}$ Involvement of extremities has the worst outcomes, especially when the lesions cross the joint, since there is a potential for joint contracture and associated limb shortening.

Previously reported studies show that localized scleroderma usually has its onset during later childhood. ${ }^{8-12}$ Based on our literature search, congenital presentation of localized scleroderma is rare. In the current study, we report a child with localized scleroderma-related presentations occurring at birth.

\section{Case Presentation}

A 2-day-old male neonate presented with a firm, erythematous, and slightly pigmented plaque on his left thigh, leading to a change in the diameter of the affected foot and contracture of the left knee. He was referred to an orthopedist when he was 3 months old with a suspicion of arthrogryposis. During this time, the lesion had progressed linearly from the top of the thigh to the leg. Preclinical assessments including the magnetic resonance imaging (MRI), ultrasonography, and electromyography (EMG) nerve conduction velocity test were normal.

Subsequently, due to the lack of diagnosis and pattern of skin involvement, he was sent to our referral rheumatology

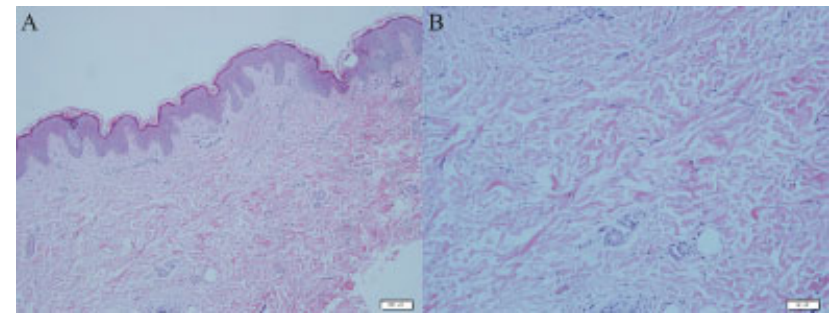

Fig. 1 Pathological view showing mild acanthosis and collagen bundles $(A: \times 100$. B: $\times 200)$.

clinic at the age of 7 months. We considered metabolic disorders as the main differential diagnosis based on the age of the patient and skin stiffness. He had normal growth and development, as well as clinical examination except for skin involvement in the leg and limited range of motion in the knee. His thyroid tests, and urine and blood high-performance liquid chromatography assay were normal. Other laboratory tests, such as complete blood cells and erythrocyte sedimentation rate were normal, and antinuclear antibodies (ANAs), antitopoisomerase I, and rheumatic factor $(\mathrm{RF})$, were negative. There were no pathological findings during his ophthalmological consultation. Skin biopsy, taken at the age of 9 months, showed mild acanthosis and collagen bundles, which replaced the fat around the sweat glands (-Fig. 1). Adnexal structures showed atrophy that was compatible with scleroderma (morphea). In immunofluorescent studies, immunoglobulin (Ig)G, IgA, IgM, and C3 were reported normal. A final diagnosis of localized scleroderma was made.

Based on the clinical features and histological pattern, a diagnosis of congenital localized scleroderma (CLS) was made. At the age of 9 months, treatment with oral prednisolone $(1 \mathrm{mg} / \mathrm{kg})$, oral methotrexate (MTX) $\left(10 \mathrm{mg} / \mathrm{m}^{2}\right)$, and colchicine $(0.25 \mathrm{mg}$ daily) was started. Progression of the skin lesion stopped after 3 months of treatment. Subsequently, corticosteroids were tapered over 6 months, while MTX and colchicine were continued for 2 years. According to the patient's age, rehabilitation program was initiated after the acute phase of the disease. Currently, due to a lack of progression in the size of the lesion, treatment has been discontinued since the past 4 years. He is now 8 years old, and the involved leg shows significant skin and subcutaneous atrophy as compared with other leg, resulting in diameter discrepancy between the two legs (-Figs. 2 and $\mathbf{3}$ ).

\section{Discussion}

Linear scleroderma is an autoimmune condition that affects the dermis and subcutaneous tissue. ${ }^{13}$ It is the most common subtype of scleroderma in childhood. ${ }^{4}$ The etiology of this disorder is unknown. Infection, trauma, radiation, and febrile illness can trigger scleroderma. ${ }^{13}$ Serological abnormalities are uncommon in children. ${ }^{13}$ When the changes are only seen in the skin, localized scleroderma is suspected. Although linear scleroderma is most often a benign disease, it may be exceptionally accompanied by the involvement of multiple organs, mainly the musculoskeletal and 

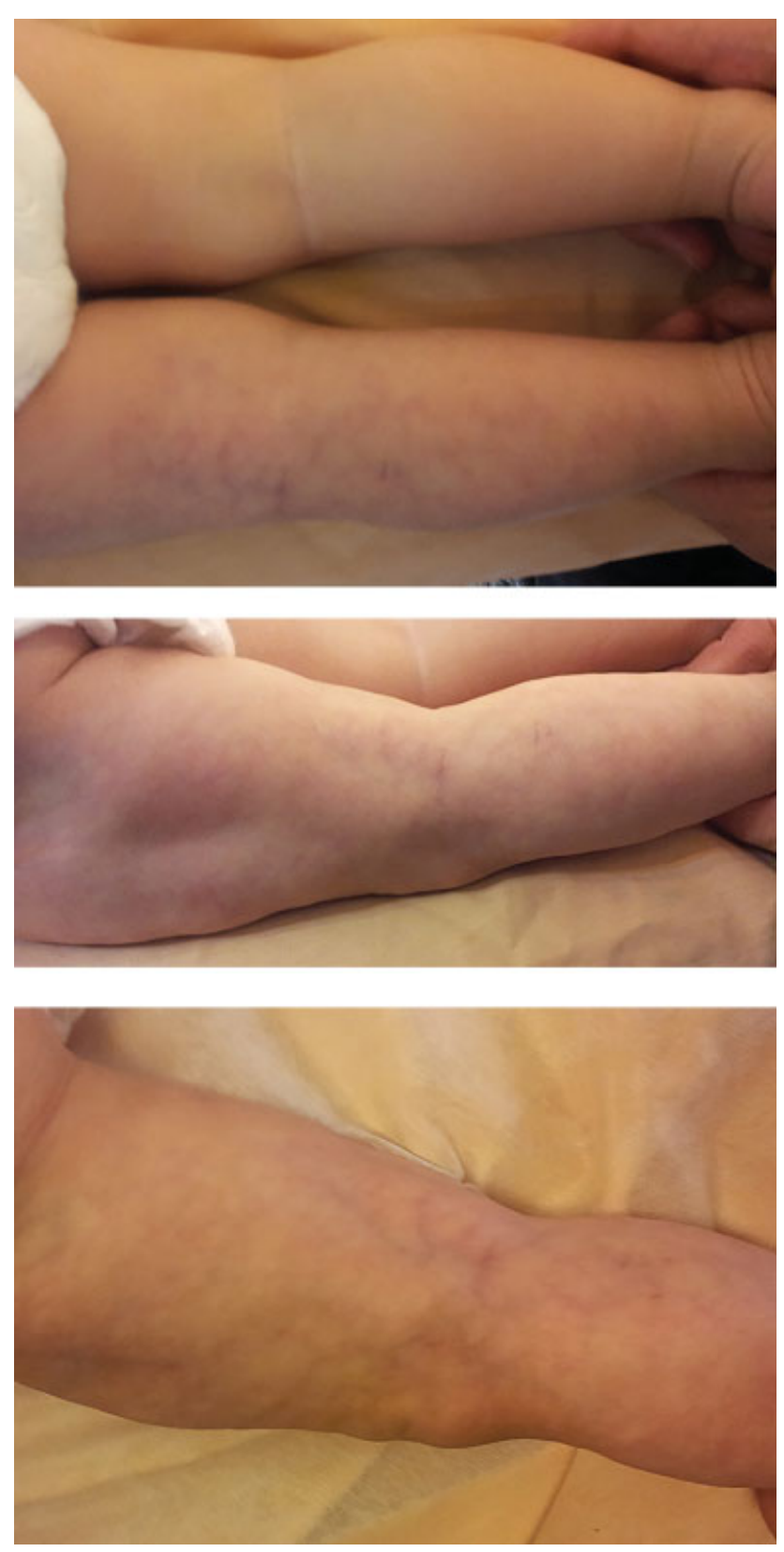

Fig. 2 Dissimilarity of the lower limbs in different views at 10 months of age.

neurological systems. ${ }^{14,15}$ In these cases, other differential diagnoses should be considered.

CLS is rare or underestimated, and it only appears with erythematous fibrotic lesions with waxy induration and firmness that is hypo- or hyperpigmented. ${ }^{4}$ The erythematous lesion is the most common manifestation of CLS, ${ }^{4}$ similar to what was seen in our patient. The patient did not have any remarkable familial history, and his mother had no history of disease or drug use during pregnancy. Involvement of the face, known as "en coup de sabre," is the most common manifestation of CLS at birth. However, our patient had exclusive limb involvement. Biopsy of the affected skin demonstrated obvious swollen collagen fibers under the thickened dermis, with depots of hyaluronic acid.

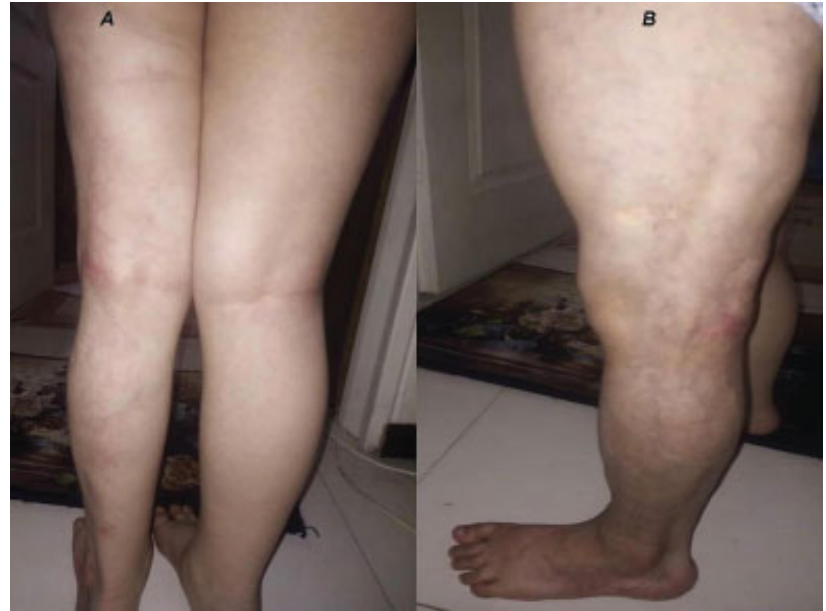

Fig. 3 Dissimilarity of the lower limbs at 7 years of age.

Histopathological examinations cannot differentiate between localized scleroderma and systemic sclerosis, and the diagnosis and differentiation of these two diseases are based on a comprehensive examination of clinical symptoms. Localized scleroderma is characterized by the absence of sclerodactyly, the Raynaud's phenomenon, and capillary changes in the nail. Furthermore, even when patients with localized scleroderma commonly have nonspecific systemic symptoms as well as the presence of autoantibodies, the typical features of systemic sclerosis visceral involvement are absent. ${ }^{16}$

Diagnosis of congenital morphea is usually made by clinical examination and confirmed by a skin biopsy. However, examination of serum markers, including ANA, RF, antihistone antibody, and double-stranded DNA may help diagnose the disease as well as determine its severity. ${ }^{6}$ Additional assessment such as ultrasonography or MRI confirms the atrophy and sclerosis of the skin or subcutaneous tissue in morphea. Laboratory studies for neuromuscular disorders may also be useful. Nerve conduction and EMG studies are complementary to the physical examination and help guide diagnostic studies such as muscle and nerve biopsies, and molecular genetic studies. The important feature pertaining to the diagnosis of this disease is delay caused by the lack of knowledge about the congenital presentation of this disease and its initial morphology being similar to other skin lesions such as port-wine stains and nevus simplex. ${ }^{4,17}$

All diagnostic information should be interpreted not in isolation but by taking into consideration the relevant historical information, family history, physical examination findings, laboratory data, electrophysiologic findings, pathologic findings, as well as molecular genetic findings if obtained.

The differential diagnoses of CLE are salmon patch (birthmark caused by expansion of capillaries), Texier's disease (pseudosclerodermatous reaction occurring after injection of vitamin K), skin infections such as cellulitis, giant congenital nevus (dark-colored patch of skin present at birth), portwine stain (discoloration of the skin due to vascular 
anomaly), stiff skin syndrome (characterized by hardness and thickness of the skin in an infant), café-au-lait spots, and nevus simplex. ${ }^{4}$

Treatment varies depending on the subtype and severity. Topical corticosteroids and phototherapy with ultraviolet light A alone or in combination with MTX are useful for treatment of superficial morphea. For lesions with involvement of the deep tissues, using MTX and corticosteroids is recommended. ${ }^{18,19}$ Other therapeutic options include topical calcineurin inhibitors, mycophenolate mofetil, biological drugs (e.g., tocilizumab, abatacept, tofacitinib), intravenous IG, surgical procedures, autologous transplants of adipose tissue, injections of botulinum toxin and hyaluronic acid. However, due to its rarity, it has not been possible to investigate whether these treatments improve outcomes in congenital morphea.

\section{Conclusion}

Localized scleroderma in early infancy is a rare disease, but it may be congenital scleroderma and should be considered as a differential diagnosis in infants with erythematous and firm lesion on their body at birth because early treatment can prevent complications in future.

\section{Authors' Contributions}

E.H.M. performed data gathering and drafting of the manuscript. M.S. performed pathology study and contributed to interpretation of the pathologic findings. V.Z. provided the concept and case of need for the survey, clinical expertise, and interpretation of the clinical data and critical revision of the final draft of manuscript. All authors read and approved the final version of the manuscript.

\section{Informed Consent}

Informed consent was obtained from the patient's parent.

\section{Funding}

None.

\section{Conflict of Interest}

None declared.

\section{References}

1 Altan G, Kose TE, Erdem TL, Ozcan I. Scleroderma: a case report. J Istanb Univ Fac Dent 2015;49(02):31-34
2 Torok KS. Pediatric scleroderma: systemic or localized forms. Pediatr Clin North Am 2012;59(02):381-405

3 Zulian F. Scleroderma in children. Pediatr Clin North Am 2005;52 (02):521-545, vii

4 Zulian F, Vallongo C, de Oliveira SK, et al. Congenital localized scleroderma. J Pediatr 2006;149(02):248-251

5 Peterson LS, Nelson AM, Su WP. Classification of morphea (localized scleroderma). Mayo Clin Proc 1995;70(11):1068-1076

6 Zulian F, Athreya BH, Laxer R, et al; Juvenile Scleroderma Working Group of the Pediatric Rheumatology European Society (PRES) Juvenile localized scleroderma: clinical and epidemiological features in 750 children. An international study. Rheumatology (Oxford) 2006;45(05):614-620

7 Christen-Zaech S, Hakim MD, Afsar FS, Paller AS. Pediatric morphea (localized scleroderma): review of 136 patients. J Am Acad Dermatol 2008;59(03):385-396

8 Uziel Y, Krafchik BR, Silverman ED, Thorner PS, Laxer RM. Localized scleroderma in childhood: a report of 30 cases. Semin Arthritis Rheum 1994;23(05):328-340

9 Vancheeswaran R, Black CM, David J, et al. Childhood-onset scleroderma: is it different from adult-onset disease. Arthritis Rheum 1996;39(06):1041-1049

10 Marzano AV, Menni S, Parodi A, et al. Localized scleroderma in adults and children. Clinical and laboratory investigations on 239 cases. Eur J Dermatol 2003;13(02):171-176

11 Bodemer C, Belon M, Hamel-Teillac D, et al. [Scleroderma in children: a retrospective study of 70 cases]. Ann Dermatol Venereol 1999;126(10):691-694

12 Christianson HB, Dorsey CS, Kierland RR, O'Leary PA. Localized scleroderma; a clinical study of two hundred thirty-five cases. AMA Arch Derm 1956;74(06):629-639

13 Dasgupta MK, Patra C, Sarkar S, Das S. Pansclerotic morphea: a male child with hemiatrophy of lower limb. Indian Dermatol Online J 2014;5(02):170-172

14 Zulian F, Vallongo C, Woo P, et al; Juvenile Scleroderma Working Group of the Pediatric Rheumatology European Society (PRES) Localized scleroderma in childhood is not just a skin disease. Arthritis Rheum 2005;52(09):2873-2881

15 Gorkiewicz-Petkow A, Kalinska-Bienias A. Systemic involvement in localized scleroderma/morphea. Clin Dermatol 2015;33(05): 556-562

16 Fett N, Werth VP. Update on morphea: part I. Epidemiology, clinical presentation, and pathogenesis. J Am Acad Dermatol 2011;64(02):217-228, quiz 229-230

17 Pickert AJ, Carpentieri D, Price H, Hansen RC. Early morphea mimicking acquired port-wine stain. Pediatr Dermatol 2014;31 (05):591-594

18 Mansour M, Liy Wong C, Zulian F, et al. Natural history and extracutaneous involvement of congenital morphea: multicenter retrospective cohort study and literature review. Pediatr Dermatol 2018;35(06):761-768

19 Marrani E, Foeldvari I, Lopez JA, Cimaz R, Simonini G. Comparing ultraviolet light A photo(chemo)therapy with methotrexate protocol in childhood localized scleroderma: evidence from systematic review and meta-analysis approach. Semin Arthritis Rheum 2018;48(03):495-503 